\title{
A VARIABLE EXPONENT BOUNDEDNESS OF THE STEKLOV OPERATOR
}

\author{
YUSUF ZEREN
}

Abstract. In this paper, a sufficiency condition for boundedness of the Steklov operator

$$
S_{h} f(x)=\frac{1}{h} \int_{x}^{x+h} f(t) d t, \quad h>0
$$

has been proved in variable exponent Lebesgue space $L^{p(.)}(0, \infty)$. Here an infinite interval $(0, \infty)$ has been considered with a new decay condition on infinity. A finite interval $[0,2 \pi]$ case with a local log- regularity condition has been studied previously in order to be applied on approximation problem.

Mathematics subject classification (2020): 41A25, 41A65, 65R10, 46E30.

Keywords and phrases: Steklov's operator, variable exponent, uniform boundedness.

\section{REFERENCES}

[1] D. Cruz-Uribe And A. Florenzia, Variable Lebesgue Spaces. Foundations and Harmonic Analysis, Applied and Numerical Harmonic Analysis, Birkhauser/Springer, Heidelberg, 2013.

[2] L. Diening, P. Harjulehto, P. Hasto And M. RuZickA, Lebesgue and Sobolev spaces with variable exponents, Lecture Not. in Math., Springer, Heidelberg, 2017; 2011.

[3] L. Diening, P. Harjulehto, P. Hasto, Y. Mizuta and T. Shimomura, Maximal functions in variable exponent spaces: Limiting cases of the exponent, Ann. Acad. Sci. Fen. Math. 34, 1 (2009), 503-522.

[4] L. Diening And S. Samko, Hardy inequality in variable exponent Lebesgue spaces, Frac. Calc. Appl. Anal. 10, 1 (2007), 1-17.

[5] D. E. Edmunds And A. NeKvinda, Averaging operators on lpn and Lp $(x)$, Math. Inequal. Appl. 5, 2 (2002), 235-246.

[6] A. GUVEn AND D. M. IsRAFILOV, Trigonometric approximation in generalized Lebesgue spaces $L^{p(x)}$, J. Math. Ineq. 4, 2 (2010), 285-299.

[7] X. Fan And D. ZhaO, On the space $L^{p(x)}(\Omega)$ and $W^{m, p(x)}(\Omega)$, J. Math. Anal. Appl. 263, 2 (2001), 424-446.

[8] V. KokilashVili, A. Meskhi, S. SAmko, And H. Rafeiro, Integral operators in non-standard function spaces: Operator Theory: Advances and Applications, Birkhäuser, Basel, 2016.

[9] O. KovaciK And J. Rakosnik, On spaces $L^{p(x)}$ and $W^{k, p(x)}$, Czechoslovak Math. J. 41, 116 (1991), 592-618.

[10] F. I. Mamedov, On Hardy type inequality in variable exponent Lebesgue space $L^{p(\cdot)}(0,1)$, Azerbaijan J. Math. 2, 1 (2012), 90-99.

[11] F. I. MAMEDOV AND A. HARMAN, On the removability of isolated singular points for degenerating nonlinear elliptic equations, Nonlinear Analysis: Theory, Meth., Appl. 71, 12 (2009), 6290-6298.

[12] F. I. Mamedov And Y. ZEREN, On a two-weighted estimation of maximal operator in the Lebesgue space with variable exponent, Annali di Matem. Applic. 190, 2 (2011), 263-275.

[13] A. NEKVINDA, A note on one-sided maximal operator in $L_{p(.)}(R)$, Math. Inequal. Appl. 13, 4 (2010), 887-897. 
[14] V. D. RAdulescu AND D. RePovs, Partial differential equations with variable exponents: Variational methods and qualitative analysis, Monographs and Research Notes in Mathematics, CRC press, Boca Raton, FL, 2015.

[15] I. I. SHARAPUDINOv, On the uniform boundedness in $L^{p}(p=p(x))$ of some families of convolution operators, (Russian) Mat. Zametki; translation in Math. Notes 59, 2; 1-2 (1996), 291-302, 320; 205212.

[16] I. I. SHARAPUDINOV, Some problems in approximation theory in the spaces $L^{p(x)}(E)$, by trigonometric polynomials, (Russian) Anal. Math. 33, 2 (2007), 135-153.

[17] I. I. SHARAPUDINOV, Approximation of functions in $L^{p(x)}$ by trigonometric polynomials, Izv. RAN, Ser. Math. 77, 2 (2013), 407-434. 